\title{
Stability of maximal hypersurfaces in spacetimes: new general conditions and application to relevant spacetimes
}

\author{
Daniel de la Fuente*, Rafael M. Rubio ${ }^{\dagger}$ and Juan J. Salamanca** \\ * Departamento de Geometría y Topología, \\ Universidad de Granada, 18071 Granada, Spain \\ E-mails: delafuente@ugr.es, jjsalamanca@ugr.es \\ $\dagger$ Departamento de Matemáticas, \\ Universidad de Córdoba, 14071 Córdoba, Spain \\ E-mail: rmrubio@uco.es
}

\begin{abstract}
We obtain new simple sufficient conditions to ensure the stability and strong stability of maximal hypersurfaces (without boundary) immersed in an arbitrary spacetime. Via these conditions, we find applications to the study of maximal hysurfaces in spatially open and closed spacetimes, which admits an infinitesimal causal symmetry, for instance pp-wave, stationary and generalized Robertson-Walker espacetimes.
\end{abstract}

Keywords: Stable maximal hypersurfaces, infinitesimal conformal symmetry, stationary spacetime, pp-wave spacetime, generalized Robertson-Walker spacetime.

MSC 2010: 53C50, 53C42, 53C 80.

\section{Introduction}

Over the last decades, maximal hypersurfaces in spacetimes have attracted a real interest from both physical and mathematical points of view. The relevance of maximal hypersurfaces in General Relativity is justified for several reasons (see [22] for details). Among them, it should be emphasized that this class of hypersurfaces has an important role in the analysis of the Cauchy problem with the purpose of dealing with simpler constraint equations or solving them, [12], [13], [14], [15, Chap. VI], [33]. Each maximal hypersurface can describe, in some relevant cases, the transition between the expanding and contracting phases of a relativistic universe. Moreover, the existence of constant mean curvature (and in particular maximal) hypersurfaces is necessary for the study of the structure of singularities in the space of solutions to the Einstein equations. On the other hand, its presence may provide information about the existence of past or future singularities [8]. Also, the deep understanding of this kind of hypersurfaces is essential to prove the positivity of the gravitational mass. They are also

\footnotetext{
${ }^{*}$ The three authors are partially supported Spanish MINECO and ERDF proyect MTM2013-47828-C2-1-P and by the Junta de Andalucía Regional Grant P09-FQM-4496.
} 
interesting for Numerical Relativity, where maximal hypersurfaces are used to integrate forward in time. Finally, we mention that in asymptotically flat spacetimes, frequently maximal hypersurfaces produce a foliation of the spacetime, defining a time function [22].

From a mathematical point of view, a maximal hypersurface is (locally) a critical point for a natural variational problem, namely, the volume functional (see, for instance, [7]). So, it is necessary to study the maximal hypersurfaces of a spacetime in order to understand its structure [6].

On the other hand, maximal hypersurfaces are also interesting because of their nice CalabiBernstein-type properties. In the history of research on maximal hypersurfaces, a striking fact was the discovery of new nonlinear elliptic problems. In fact, a function defining a maximal graph in the $(n+1)$-dimensional Lorentz-Minkowski spacetime $\mathbb{L}^{n+1}$, with $n \geq 2$, satisfies an elliptic second order PDE similar to the equation of minimal graphs in Euclidean space $\mathbb{R}^{n+1}$, but with a new and surprising behavior for its entire solutions: The only entire solutions to the maximal hypersurface equation in $\mathbb{L}^{n+1}$ are the affine functions defining spacelike hyperplanes. This theorem was previously proved by Calabi [9] for $n \leq 3$ and later extended for arbitrary dimension by S.Y. Cheng and S.T. Yau [11]. This result is known as Calabi-Bernstein theorem. Recall that the Bernstein theorem for minimal graphs in the Euclidean space $\mathbb{R}^{n+1}$, holds only for $n \leq 7$, [29]. An important auxiliary result in [11] was the introduction of a new tool, the so-called Omori-Yau generalized maximum principle [24], [32]. By means of this technique, many uniqueness results were obtained. For instance, S. Nishikawa [23] proved that a complete maximal hypersurface in a locally symmetric Lorentzian manifold whose Ricci tensor satisfies a natural assumption on timelike tangent vectors, must be totally geodesic (Remark 5.10).

In this paper, we are interested in a remarkable subfamily of maximal hypersurfaces: the stable maximal hypersurfaces. This subfamily falls between that of the volume-maximizing spacelike hypersurfaces and that of the totality of all maximal hypersurfaces. There are various notions of stability, but basically a stable maximal surface is volume-minimizing relative to nearby spacelike hypersurfes with the same boundary.

Recall that a spacelike hypersurface $S$ in a spacetime is maximal if its mean curvature vanishes. Since maximality is equivalent to the vanishing of the first variation of volume under spacelike deformations of the hypersurface living the boundary fixed, then maximality is a necesary condition for a spacelike hypersurface to be volumen maximizing. However, if for some deformation the second variation of volume is positive, then there are nearby spacelike hypersurfaces of greater volumen and the maximal hypersursurface is called unstable. For instance, the equator of De Sitter spacetime is a hypersurface with null mean curvature but which is a saddle point of the volume functional.

In this work we say that a maximal hypersurface is stable, if it is not unstable, i.e., if its second variation of volumen is non-positive. When this last quantity is negative, we say that the maximal hypersurface is strongly stable. In this case, the maximal hypersurfce is in fact, volume-maximizing relative relative to nearby spacelike hypersurfes with the same boundary.

If we consider an open (without boundary) maximal hypersurface, it is called stable (resp. strongly stable) if $S$ is stable (resp. strongly stable) for every relative compact domain in $S$. Note that that when $S$ is a compact without boundary maximal hypersurface, the variation can be defined on all $S$.

From a physical point of view, a complete maximal strongly stable hypersurface typically describe the turn around epoch which separates the expansion from the recontraction phase in a sapcetime. Taking into account this interpretation, Brill and Flaherty [8] study strong 
stability of compact (without boundary) maximal hypersurfaces immersed in certain spacetimes (see also, [19]). Recall that a spacetime is said spatially closed if it admits a complete compact spacelike hypersurfaces. On the contrary, the spacetime is called spatially open.

In this paper, we deal with stability and and strong stability of maximal hypersurfaces (without boundary) immersed in spacetimes. So, we establish a sufficient condition (see, Lemma (1) in Section 3), which assures stability or strong stability of maximal hypersurfaces in an arbitrary spacetime. In Section 4, as a application of the Lemma (1), we obtain several results of stability and strongly stability for arbitrary spacetimes, which obey an usual energy condition, in particular stability in spacetimes, which satisfies the Einstein vacuum equations is studied.

On the other hand, we also deal with stable and strongly stable maximal hypersurfaces in a wide class of spacetime, wich present a causal infinitesimal symmetry. The notion of symmetry is basic in physics. In general relativity, symmetry is usually based on the assumption of the existence of a one-parameter group of transformations generated by a Killing or, more generally, conformal vector field. In fact, an usual simplification for the search of exact solutions to the Einstein equation is to assume the existence a priori of an infinitesimal symmetry (see [17] and [18] for instance). Although the same causal character for the infinitesimal symmetry is not always assumed, the timelike, or causal in general, is a natural choice. Moreover, this choice is supported by very well-known examples of exact solutions (stationary, pp-waves, Robertson-Walker spacetimes,...). A complete general approach to symmetries in general relativity can be found in [34]. Theorem 6, in Section 4 provides a clear and simple condition on the conformal factor to assure the stability or strongly stability of a maximal hypersurface inmersed in a spacetime with an infinitesimal causal symmetry.

Finaly, Section 5 is devoted to analyse the stability and strong stability of maximal hypersurfaces in a relevant family of cosmological models.

\section{Preliminaries}

Let $(M, \bar{g})$ be a $(n+1)$-dimensional spacetime, that is, $(M, \bar{g})$ is a time-oriented (connected) Lorentzian manifold. Given an $n$-dimensional manifold $S$, an immersion $x: S \rightarrow M$ is said to be spacelike if the Lorentzian metric given by $\bar{g}$ induces, via $x$, a Riemannian metric $g$ on $S$. In this case, $S$ is called a spacelike hypersurface. Since the Lorentzian manifold $(M, \bar{g})$ is timeoriented, we can take, for each spacelike hypersurface $S$ in $M$, the vector field $N \in \mathfrak{X}^{\perp}(S)$ as the only globally defined unitary timelike vector field normal to $S$ in the time-orientation of the spacetime.

Let us represent by $\bar{\nabla}$ and $\nabla$ the Levi-Civita connections of the metric $\bar{g}$ and $g$, respectively. The Gauss and Weingarten formulas of $S$ are respectively

$$
\begin{aligned}
\bar{\nabla}_{X} Y & =\nabla_{X} Y-g_{S}(A X, Y) N, \\
A X & =-\bar{\nabla}_{X} N,
\end{aligned}
$$

for all $X, Y \in \mathfrak{X}(S)$, where $A$ is the shape operator associated to $N$. Recall that the mean curvature function relative to $N$ is $H:=-(1 / n) \operatorname{trace}(A)$. The mean curvature is zero if and only if the spacelike hypersurface is, locally, a critical point of the $n$-dimensional area functional for compactly supported normal variations. A spacelike hypersurface with $H=0$ is called a maximal hypersurface. 


\section{$3 \quad$ Sep up}

Let $x: S^{n} \rightarrow M^{n+1}$ be a spacelike hypersurface immersed in a spacetime $(M, \bar{g})$. We denote by $g$ the induced metric on $x(S) \equiv S$. Let $N$ be the future-directed unit normal vector field to $S$ and let $A$ be the shape operator associated to $N$.

Given a normal variation of $S$, the corresponding variational vector field along $S$ is represented by $\phi N$, where $\phi$ is a function on $S$. The variation have compact support when the function $\phi$ have compact support on $S$, i.e., $\phi \in C_{0}^{\infty}(S)$.

Recall that the volume of a compact set $\Omega \subset S$ is given by

$$
\operatorname{Vol}(\Omega)=\int_{\Omega} \omega_{S}
$$

where $\omega_{S}$ is the induced Riemannian volume element of $S$.

The second variation of the volume functional of the maximal hypersurface $\Omega$, with boundary, is given by (see [4], for instance),

$$
\left.\frac{d^{2}}{d s^{2}}\right|_{s=0} \operatorname{Vol}\left(\Omega_{s}\right)=\int_{S}\left[\Delta \phi-\overline{\operatorname{Ric}}(N, N) \phi-\operatorname{trace}\left(A^{2}\right) \phi\right] \phi \omega_{S},
$$

where $\operatorname{supp}(\phi) \subset \Omega$.

Taking (3) into account, it useful to define the quadratic form

$$
Q(\phi, \phi)=\int_{S}\left[\Delta \phi-\left(\overline{\operatorname{Ric}}(N, N)+\operatorname{trace}\left(A^{2}\right)\right) \phi\right] \phi \omega_{S}, \quad \phi \in C_{0}^{\infty}(S),
$$

where $\overline{\text { Ric }}$ denotes the Ricci tensor of $M$ and $\Delta$ the Laplacian with respect to the induced metric $g$. A maximal hypersurface (without boundary) $S$ is called stable if $Q(\phi, \phi) \leq 0$ for all compact supported function $\phi$ on $S$. Analogously, the maximal hypersurface will be strongly stable if $Q(\phi, \phi)<0$ for all function $\phi$ with compact support and non identically null. For the case of a minimal surface immersed in certain Riemannian 3-manifolds, Barta [5] proved that the existence of a positive function $u$ defined on a minimal hypersurface such that $L u=0$, being $L$ the corresponding Jacobi operator for the Riemannian case, ensures the stability of the minimal hypersurface (see also, [16]).

Next, we obtain a stronger analogous result for maximal hypersurfaces in the Lorentzian ambient, via its respective Jacobi operator,

$$
L u=\Delta u-\left[\overline{\operatorname{Ric}}(N, N)+\operatorname{trace}\left(A^{2}\right)\right] u .
$$

Lemma 1 Let $S$ be a maximal hypersurface in a spacetime $(M, \bar{g})$. If there exists a positive function $u \in C^{\infty}(S)$ satisfying $L u \leq 0$ (resp. Lu $<0$ ), then $S$ is stable (resp. strongly stable).

Proof. Assume that there exists such a positive function $u$. Let $\phi=\varphi u$ be an arbitrary compact supported function, for a certain $\varphi \in C_{0}^{\infty}(S)$.

If we denote by $\nabla$ the gradient the gradient operator of $S$ and using

$$
\int_{S} \phi \Delta \phi \omega_{S}=\int_{S}\left(\varphi^{2} u \Delta u+\varphi u^{2} \Delta \varphi+2 \varphi u g(\nabla \varphi, \nabla u)\right) \omega_{S}
$$


we obtain,

$$
\begin{aligned}
Q(\phi, \phi) & =\int_{S}\left[\varphi^{2} u\left(\Delta u-\left(\operatorname{trace}\left(A^{2}\right)+\overline{\operatorname{Ric}}(N, N)\right) u\right)+\varphi u^{2} \Delta \varphi+2 \varphi u g(\nabla \varphi, \nabla u)\right] \omega_{S} \\
& \leq \int_{S}\left(\varphi u^{2} \Delta \varphi+2 \varphi u g(\nabla \varphi, \nabla u)\right) \omega_{S}=\int_{S}\left(\frac{1}{2} g\left(\nabla \varphi^{2}, \nabla u^{2}\right)+\varphi u^{2} \Delta \varphi\right) \omega_{S} \\
& =-\int_{S}|\nabla \varphi|^{2} u^{2} \omega_{S} \leq 0 .
\end{aligned}
$$

In the last step we have taken into account that

$$
\operatorname{div}\left(u^{2} \nabla \varphi^{2}\right)=g\left(\nabla \varphi^{2}, \nabla u^{2}\right)+u^{2} \Delta \varphi^{2}=g\left(\nabla \varphi^{2}, \nabla u^{2}\right)+2 u^{2}\left(\varphi \Delta \varphi+|\nabla \varphi|^{2}\right) .
$$

On the other hand, if $\phi$ is not zero and $L u<0$, then $\int_{S}\left[\varphi^{2} u\left(\Delta u-\left(\operatorname{trace}\left(A^{2}\right)+\overline{\operatorname{Ric}}(N, N)\right) u\right)\right] \omega_{S}<$ 0 and $Q(\phi, \phi)<0$.

Remark 2 Observe that in the previous result, if the function $u$ on the maximal hypersurface $S$ satisfies $L u \leq 0$ and the points $p \in M$ such that $L u(p)=0$ are isolated points, we also can assert that $S$ is strongly stable.

\section{$4 \quad$ First results}

As a direct consequence of the Lemma (1), we can obtain a first general result.

Recall that a spacetime obeys the Timelike Convergent Condition (TCC) if its Ricci tensor satisfies

$$
\overline{\operatorname{Ric}}(Z, Z) \geq 0,
$$

for all timelike vector $Z \in \mathfrak{X}(M)$. It is normally argued that TCC is the mathematical translation that gravity, on average, attracts, [28].

On the other hand, a spacetime is said to have non-vanishing matter fields, or obeys the ubiquitous energy condition (UEC) [30], if $\overline{\operatorname{Ric}}(Z, Z)>0$, for all timelike tangent vector $Z$. This last energy condition is stronger than TCC and roughly means a real presence of matter at any point of the spacetime.

The following theorem assures stability and strong stability under the previous energy conditions.

Theorem 3 Every maximal hypersurface immersed in a spacetime satisfying the TCC (resp. $U E C)$ is stable (resp. strongly stable).

Proof. It is enough to apply Lemma (1) to the constant function $u \equiv 1$ defined on the maximal hypersurface.

Remark 4 As a direct consequence of the previous Theorem, a complete maximal hypersurface in a spacetime, which satisfies the UEC, describe the turn around epoch, which separates the expansion from the recontraction phase. 
On the other hand, when the spacetime is a vacuum solution, i.e., a time-oriented Lorentzian manifold whose Einstein tensor vanishes identically, it is well-known that Einstein equation is equivalent to:

$$
\overline{\operatorname{Ric}}=0 .
$$

The following reslt extends [8, Th. 4.1] to spatially open spacetimes.

Corollary 5 Every complete maximal hypersurface $S$ immersed in a spacetime which satisfies the Einstein vacuum equations must be stable. Moreover, either $S$ is strongly stable or is a totally geodesic spacelike hypersurface.

\subsection{Stabilility of maximal hypersurfaces in spacetimes with a causal in- finitesimal symmetry}

Recall that vector field $K \in \mathfrak{X}(M)$, defined on a spacetime $(M, \bar{g})$ is conformal if there exists a smooth function $\rho$ (conformal factor) such that

$$
\mathcal{L}_{K} \bar{g}=2 \rho \bar{g},
$$

where $\mathcal{L}$ denotes the Lie derivative.

Equation (6) is equivalent to

$$
\bar{g}\left(\bar{\nabla}_{X} K, Y\right)+\bar{g}\left(\bar{\nabla}_{Y} K, X\right)=2 \rho \bar{g}(X, Y) .
$$

It is well-known that a vector field $K$ is conformal if and only if the stages $\phi_{s}$ of all its (local) flows are conformal maps. When $\rho$ vanishes identically, $K$ is a Killing vector field, and the stages $\phi_{s}$ of all its (local) flows are isometries (see, [25]). In this work, we are interested in that symmetries given by a causal conformal vector field.

From now on, we assume that the spacetime $(M, \bar{g})$ admits a causal or timelike conformal vector field $K$, according to each case. The existence of a timelike comformal vector field is a natural choice, since the integral curves of such a timelike infinitesimal symmetry provide a privileged class of observers or test particles in the spacetime (see, [17], [10]).

On the other hand, the existence of a timelike Killing vector field on a spacetime $(M, \bar{g})$ is specially useful to study its geometry. It is well-known that around each point $p$, there exists coordinates $\left(t, x^{1}, \ldots, x^{n}\right)$ such that $K=\partial_{t}$ and all the component $\bar{g}_{i j}$ of the metric are independent of $t$; this justifies the name stationary for the spacetime. The observers along $K$ (stationary observers) not only see a non-changing metric but also find a constant $E=\bar{g}\left(\partial_{t}, \gamma^{\prime}\right)$ for any geodesic $\gamma$. Thus, photons and freely falling particles has constant energy $E$ for these observers. When the timelike Killing vector field $K$ is irrotational, i.e. the orthogonal distribution $K^{\perp}$ is involutive, then a local warped product structure appears (see, [25, Chap. 12]) and there exists coordinates with $\bar{g}_{0 i}=0$, for $i=1, \ldots, n$, where we denote $t:=x_{0}$. In this case, the spacetime is said static and the observers along $K$ measure a metric with no cross terms between space and time. Since the orthogonal distribution is involutive, the Frobenious theorem (see, [31]) assures the existence of restspaces for every observer along $K$ and the local flow of $K$ keeps the restspaces.

A more general family is given when the spacetime admits a timelike conformal vector field. In this case the spacetime is called conformally stationary (see, [3], [17]). If $K$ is a 
timelike conformal vector field and its corresponding conformal factor is constant on all the spacetime, then $K$ is said to be homothetical (see, [17]).

Another important case arises when $K$ is a lightlike and closed vector field (and consequently parallel). Such a spacetime is known as pp-wave spacetime (see, [21]). It models electromagnetic or gravitational radiation moving at the speed of light. Its recent interest can be explained by its applications to string theory and gravitational waves.

Let $S$ be an immersed maximal hypersurface in a spacetime $(M, \bar{g})$, which admits a causal conformal vector field $K \in \mathfrak{X}(M)$, with conformal factor $\rho \in C^{\infty}(M)$.

Consider the distinguished function $u:=-\bar{g}(K, N)$ on $S$, where $N$ denotes the futuredirected normal unitary vector field on the maximal hypersurface. Let $X_{p} \in T_{p} S$ be, $p \in S$ an arbitrary tangent vector, so

$$
X_{p}(u)=-\bar{g}\left(\bar{\nabla}_{X_{p}} K, N\right)-\bar{g}\left(K, \bar{\nabla}_{X_{p}} N\right)=\bar{g}\left(\bar{\nabla}_{N} K, X_{p}\right)+\bar{g}\left(A K^{\top}, X_{p}\right),
$$

where $K^{\top}$ denotes the tangential component of $K$ along $S$, i.e., $K^{\top}=K+\bar{g}(K, N) N$. Therefore,

$$
\nabla u=A K^{\top}+\left(\bar{\nabla}_{N} K\right)^{\top}
$$

We need to apply the Jacobi operator $L$ to the function $u$. In order to perform that, we compute its Laplacian.

Given $p \in S$, making use of the exponential map, we can extend the unitary normal vector field on a tubular neighborhood $\tilde{\mathcal{U}}$ of a suitable open subset $\mathcal{U} \subset S$, with $p \in \mathcal{U}$. Taking a local orthonormal frame $\left\{E_{i}\right\}_{i=1, \ldots, n}$ around $p \in S$ and extending it on an open subset of $\tilde{\mathcal{U}}$ satisfying $\left[E_{i}, N\right]=0$ for all $i=1, \ldots, n$, we have

$$
\begin{aligned}
\Delta u & =\sum_{i}\left[g\left(\nabla_{E_{i}}\left(A K^{\top}\right), E_{i}\right)+g\left(\nabla_{E_{i}}\left(\bar{\nabla}_{N} K\right)^{\top}, E_{i}\right)\right] \\
& =\sum_{i}\left[\bar{g}\left(A E_{i}, \nabla_{E_{i}} K^{\top}\right)+\bar{g}\left(\left(\nabla_{E_{i}} A\right) K^{\top}, E_{i}\right)+\bar{g}\left(\nabla_{E_{i}}\left(\bar{\nabla}_{N} K\right)^{\top}, E_{i}\right)\right] .
\end{aligned}
$$

Now, for the first addend of (9) we have

$$
\sum_{i} \bar{g}\left(A E_{i}, \bar{\nabla}_{E_{i}} K^{\top}\right)=-\bar{g}(K, N) \operatorname{trace}\left(A^{2}\right)+\sum_{i} \bar{g}\left(A E_{i}, \bar{\nabla}_{E_{i}} K\right)
$$

On the other hand, making use of the Codazzi equation on the second addend, we obtain

$$
\sum_{i} \bar{g}\left(\left(\nabla_{E_{i}} A\right) K^{\top}, E_{i}\right)=-\sum_{i} \bar{g}\left(\overline{\mathrm{R}}\left(E_{i}, K^{\top}\right) N, E_{i}\right)+\sum_{i} \bar{g}\left(\left(\nabla_{K^{\top}} A\right) E_{i}, E_{i}\right) .
$$

Moreover, taking into account that tensor derivations commute with contractions,

$$
\sum_{i} \bar{g}\left(\left(\nabla_{K^{\top}} A\right) E_{i}, E_{i}\right)=-n \bar{g}\left(K^{\top}, \nabla H\right)
$$

Therefore, since $S$ is maximal, equation (11) can be written as

$$
\sum_{i} \bar{g}\left(\left(\nabla_{E_{i}} A\right) K^{\top}, E_{i}\right)=-\overline{\operatorname{Ric}}\left(K^{\top}, N\right) .
$$


Finally, we compute the last term of equation (9),

$$
\begin{aligned}
\sum_{i} \bar{g}\left(\nabla_{E_{i}}\left(\bar{\nabla}_{N} K\right)^{\top}, E_{i}\right) & \\
& =\sum_{i} \bar{g}\left(\bar{\nabla}_{E_{i}}\left(\bar{\nabla}_{N} K\right)^{\top}, E_{i}\right) \\
& =\sum_{i} \bar{g}\left(\bar{\nabla}_{E_{i}} \bar{\nabla}_{N} K, E_{i}\right)+\sum_{i} \bar{g}\left(\bar{\nabla}_{E_{i}} \bar{g}\left(\bar{\nabla}_{N} K, N\right) N, E_{i}\right) \\
& =\sum_{i}\left[\bar{g}\left(\overline{\mathrm{R}}\left(E_{i}, N\right) K, E_{i}\right)+\bar{g}\left(\bar{\nabla}_{N} \bar{\nabla}_{E_{i}} K, E_{i}\right)+\bar{g}\left(\bar{\nabla}_{N} K, N\right) \bar{g}\left(\bar{\nabla}_{E_{i}} N, E_{i}\right)\right] \\
& =\overline{\operatorname{Ric}}(N, K)+N(\overline{\operatorname{div}}(K))+N\left(\bar{g}\left(\bar{\nabla}_{N} K, N\right)\right)+\sum_{i} \bar{g}\left(A E_{i}, \bar{\nabla}_{E_{i}} K\right) \\
& -n \bar{g}\left(\bar{\nabla}_{N} K, N\right) H,
\end{aligned}
$$

where $\overline{\operatorname{div}}$ denotes the divergence operator of $M$ and the mean curvature.

Since, the vector field $K$ is conformal, we have

$$
\overline{\operatorname{div}}(K)=(n+1) \rho \quad \text { and } \quad \bar{g}\left(\bar{\nabla}_{N} K, N\right)=-\rho .
$$

Making use of (9), (10), (13) and (14), with $H=0$, we obtain

$$
\Delta u=-\bar{g}(K, N)\left[\operatorname{trace}\left(A^{2}\right)+\overline{\operatorname{Ric}}(N, N)\right]+n N(\rho)+2 \sum_{i} \bar{g}\left(A E_{i}, \bar{\nabla}_{E_{i}} K\right)
$$

Moreover, the last term of (15) vanishes. Indeed,

$$
\begin{aligned}
\sum_{i} \bar{g}\left(A E_{i}, \bar{\nabla}_{E_{i}} K\right) & =\sum_{i, j} \bar{g}\left(A E_{i}, E_{j}\right) \bar{g}\left(E_{j}, \bar{\nabla}_{E_{i}} K\right) \\
& =-\sum_{i, j}\left[\bar{g}\left(A E_{i}, E_{j}\right) \bar{g}\left(E_{i}, \bar{\nabla}_{E_{j}} K\right)+\rho \bar{g}\left(A E_{i}, E_{j}\right) \bar{g}\left(E_{i}, E_{j}\right)\right] \\
& =-\sum_{i}\left[\bar{g}\left(A E_{i}, \bar{\nabla}_{E_{i}} K\right)+\rho \bar{g}\left(A E_{i}, E_{i}\right)\right]
\end{aligned}
$$

where we have used that $K$ is conformal. Hence,

$$
\sum_{i} \bar{g}\left(A E_{i}, \bar{\nabla}_{E_{i}} K\right)=\frac{1}{2} \rho n H=0 .
$$

Therefgore, we obtain

$$
\Delta u=-\bar{g}(K, N)\left[\operatorname{trace}\left(A^{2}\right)+\overline{\operatorname{Ric}}(N, N)\right]+n \bar{g}(N, \bar{\nabla} \rho),
$$

or equivalently,

$$
L u=n \bar{g}(N, \bar{\nabla} \rho) .
$$

As a direct consequence of (16) we can enunciate a first result. 
Theorem 6 Let $(M, \bar{g})$ be a spacetime, which admits a causal conformal vector field $K$, with conformal factor $\rho$.

(i) If $\nabla \rho$ is a future-directed causal vector field, then every maximal hypersurface in $M$ is strongly causal.

(ii) If the vecor field $\nabla \rho$ is is at each point $p \in M$ a future-directed causal vector or the zero vector, then every maximal hypersurface in $M$ is stable.

Observe that from Remark 2 , if $\nabla \rho$ is a future-directed causal vector field, except for at most some isolated points, where the gradient is zero, then (i) in the previous theorem also holds.

In particular, from the Theorem 6 , we obtain,

Corollary 7 Let $(M, \bar{g})$ be a spacetime which admits a causal Killing vector field. Then every maximal hypersurface in $M$ is stable. Specifically, in stationary and pp-waves spacetimes every maximal hypersurface is stable.

On the other hand, from formula (16) we can also provide the following results.

Theorem 8 Let $(M, \bar{g})$ be a spacetime, which admits a causal conformal vector field such that the gradient of the conformal factor is a causal past-directed vector field, then every maximal compact hypersurface must be unstable.

Proof. It is enough to observe that $u$ is a positive compact supported function on the hypersurface satisfying $Q(u, u)>0$.

\section{$5 \quad$ Stability of maximal hypersurfaces in generalized Robertson- Walker spacetimes}

In this section, we focus on a special class of spacetimes admitting a conformal vector field, the generalized Robertson-Walker (GRW) spacetimes. This class of cosmological models are warped products $I \times_{f} F$ with base an open interval $\left(I,-d t^{2}\right)$, fiber a Riemannian manifold $\left(F, g_{F}\right)$ whose sectional curvature is not assumed to be constant and warping function $f(t)$ defined on $I$ (see, [2]). Thus, these family of spacetimes widely extend to those that are classically called Robertson-Walker (RW) spacetimes. Recall that the class of RobertsonWalker spacetimes includes the usual big-bang cosmological models, the de Sitter spacetime, the steady state spacetime, the Lorentz-Minkowsky spacetime and the Einstein's static spacetime, among others. Unlikely to these spacetimes, the GRW spacetimes are not necessarily spatially-homogeneous. Note that being spatially-homogeneous, which is reasonable as a first approximation of the large scale structure of the universe, could not be appropriate when we consider a more accurate scale. Thus, a GRW spacetime could be a suitable spacetime to model a universe with inhomogeneous spacelike geometry [26]. On the other hand, small deformations of the metric on the fiber of classical Robertson-Walker spacetimes fit into the class of GRW spacetimes. Therefore, GRW spacetimes are useful to analyze if a property of a RW spacetime $\bar{M}$ is stable, i.e. if it remains true for spacetimes close to $\bar{M}$ in a certain topology defined on a suitable family of spacetimes [20]. In fact, a deformation $s \mapsto g_{F}^{(s)}$ of the metric of $F$ provides a one parameter family of GRW spacetimes close to $\bar{M}$ when $s$ 
approaches to 0. Note also that a conformal change of the metric of a GRW spacetime with a conformal factor which only depends on $t$, produces a new GRW spacetime.

Specifically, let $\left(F, g_{F}\right)$ be an $n(\geq 2)$-dimensional (connected) Riemannian manifold, $I$ an open interval in $\mathbb{R}$ endowed with the metric $-d t^{2}$, and $f$ a positive smooth function defined on $I$. Then, the product manifold $I \times F$ endowed with the Lorentzian metric

$$
\bar{g}=-\pi_{I}^{*}\left(d t^{2}\right)+f\left(\pi_{I}\right)^{2} \pi_{F}^{*}\left(g_{F}\right),
$$

where $\pi_{I}$ and $\pi_{F}$ denote the projections onto $I$ and $F$, respectively, is called a generalized Robertson-Walker $(G R W)$ spacetime with fiber $\left(F, g_{F}\right)$, base $\left(I,-d t^{2}\right)$ and warping function $f$. Usually, the previous Lorentzian warped product is represented by $M=I \times{ }_{f} F$.

The family of spacelike hypersurfaces $\Sigma_{t}=\{t\} \times F=\{(t, p): p \in F\}, t \in I$, constitutes a foliation of $M$ by totally umbilical leaves of constant mean curvature $H=\frac{f^{\prime}(t)}{f(t)}$ that we will call spacelike slices. We will say that a spacelike hypersurface $x: S \longrightarrow M$ is contained in a slab if it is contained between two spacelike slices, that is, if the height function $\tau:=t \circ x$ is such that $\tau(S) \subseteq\left[t_{1}, t_{2}\right]$ for $t_{1}, t_{2} \in I, t_{1}<t_{2}$.

The behavior of warping function (or scale factor) has important consequences for this class of cosmological models. If for each $p \in F$, we parametrize the worldline of each galaxy $I \times\{p\}$ by $\gamma_{p}(t)=(t, p)$, since $\partial_{t}$ is the velocity of each $\gamma_{p}$, they are its integral curves. In particular, the function $t$ is the common proper time of all galaxies. Let us consider a fixed spacelike slice $\Sigma_{t}$. The distance between two galaxies $\gamma_{p}$ and $\gamma_{q}$ in $\Sigma_{t}$ is $f(t) d(p, q)$, where $d$ is the Riemannian distance in the fiber $F$. In particular, when $f$ has positive derivative (resp. negative derivative) the spaces $\Sigma_{t}$ are expanding (resp. contracting). Moreover, if $f^{\prime \prime}>0$ (resp. $f^{\prime \prime}<0$ ) the GRW spacetimes models universes in accelerated expansion (resp. contraction). On the other hand, when $f^{\prime}>0$ and $f^{\prime \prime}<0$, the spacetime time is in decelerated expansion. This is the case of the well-known Einstein-de Sitter spacetime.

In the context of GRW spacetimes, it is not difficult to see that the TCC is equivalent to

$$
f^{\prime \prime} \leq 0, \quad \operatorname{Ric}^{F}(X, X) \geq(n-1)\left(f f^{\prime \prime}-f^{\prime 2}\right) g_{F}(X, X),
$$

for all $X$ tangent to the fiber $F$, where $\operatorname{Ric}^{F}$ denotes the Ricci tensor of $\left(F, g_{F}\right)$.

On the other hand, a GRW spacetime satisfies the ubiquitous energy condition if (19) holds, with $f^{\prime \prime}<0$.

For an arbitrary spacetime a weaker energy condition is the Null Convergence Condition (NCC) which reads $\operatorname{Ric}(Z, Z) \geq 0$ for all null tangent vector $Z$. So, this energy condition only applies to light particles. It is easy to see that a GRW spacetime $M$ obeys the NCC if and only if

$$
\operatorname{Ric}^{F}-(n-1) f^{2}(\log f)^{\prime \prime} \geq 0,
$$

where $\operatorname{Ric}^{F}$ denotes the Ricci curvature of the fiber at any arbitrary direction. It is clearly that if the Ricci curvature of the fiber is positive definite and the warping function satisfies $(\log f)^{\prime \prime} \leq 0$, then the GRW spacetime obeys the NCC.

Note that $f^{\prime \prime} \leq 0$ implies $(\log f)^{\prime \prime} \leq 0$. Nevertheless, the last inequality can be compatible with certain accelerated expanding models (see, [1]). So the well-known steady state spacetime is given as a GRW spacetime with fiber $\mathbb{R}^{n}$ and warping function $f(t)=e^{t}$. 
On other hand, in a GRW spacetime there is a remarkable timelike conformal vector field, $K=f(t) \partial_{t}$, satisfying $\mathcal{L}_{K} \bar{g}=2 f^{\prime}(t) \bar{g}$. Thus, the conformal factor is given by $\rho=f^{\prime}(t)$, and

$$
\bar{\nabla} \rho=-f^{\prime \prime}(t) \partial_{t}
$$

Now, we can to state a new result in the case of GRW spacetimes.

Theorem 9 Every maximal hypersurface in a GRW spacetime, whose warping function satisfies the convexity condition $f^{\prime \prime} \leq 0$ (resp. $f^{\prime \prime}<0$ ) is stable (resp. strongly stable).

Under some natural assumptions on the convexity of the warping function and on the geometry of the spacetime, we obtain the next theorem. A technical lemma by Omori and Yau ([24], [32]) is necessary.

Lemma 10 Let $S$ be a complete Riemannian manifold whose Ricci curvature is bounded away from below and let $u: S \longrightarrow \mathbb{R}$ be a smooth function bounded from below (resp. bounded from above). Then, for each $\varepsilon>0$, there is a point $p_{\varepsilon} \in S$ such that

1. $\left|\nabla u\left(p_{\varepsilon}\right)\right|<\varepsilon$,

2. $\Delta u\left(p_{\varepsilon}\right)>-\varepsilon\left(\right.$ resp. $\left.\Delta u\left(p_{\varepsilon}\right)<\varepsilon\right)$,

3. $\inf (u) \leq u\left(p_{\varepsilon}\right)<\inf (u)+\varepsilon\left(\right.$ resp. $\left.\sup (u)-\varepsilon<u\left(p_{\varepsilon}\right) \leq \sup (u)\right)$.

Theorem 11 Let $S$ a complete maximal hypersurface in a GRW spacetime whose fiber has sectional curvature bounded from below and whose warping function satisfies $(\log f)^{\prime \prime} \leq 0$ $\left(\right.$ resp. $\left.(\log f)^{\prime \prime}<0\right)$. If $S$ lies in a slab, then it is stable (resp. strongly stable).

Proof.

Let us define the auxiliar smooth function $F(\tau)=\int_{\inf \tau}^{\tau} f(s) d s \in C^{\infty}(S)$, which is bounded because $S$ lies in a slab. A straightforward computation gives

$$
\Delta(F(\tau))=-n f^{\prime}(\tau)
$$

Taking into account [27, Lemma 7], the Ricci curvature of the maximal hypersurface $S$ is bounded from below. Making use of the Lemma 10 we have that for each $\varepsilon>0$, there exists a point $p_{\varepsilon} \in S$ such that

$$
\mid \nabla F\left(\tau\left(p_{\varepsilon}\right)\right)<\varepsilon, \quad \text { and } \quad-\varepsilon \leq \Delta(F(\tau))\left(p_{\varepsilon}\right)=-n f^{\prime}\left(\tau\left(p_{\varepsilon}\right)\right),
$$

with $\inf (F(\tau(S))) \leq F\left(\tau\left(p_{\varepsilon}\right) \leq \inf (F(\tau(S)))+\varepsilon\right.$.

Since $\inf (F(\tau(S)))=F(\inf \tau(S))$, doing $\varepsilon \rightarrow 0_{+}$we get

$$
0 \leq-n f^{\prime}(\inf \tau(S)) \quad \text { and } \quad \frac{f^{\prime}(\inf \tau(S))}{f(\inf \tau(S))} \leq 0 .
$$

Analogously, since $F(\tau)$ is bounded from above, we obtain that

$$
\frac{f^{\prime}(\sup \tau(S))}{f(\sup \tau(S))} \geq 0 .
$$


Taking into account the the function $\frac{f^{\prime}(t)}{f(t)}$ is non-increasing, we obtain that $f^{\prime}(\tau)=0$ and as a consequence $f^{\prime \prime}(\tau) \leq 0$.

Note that under the assumption $(\log f)^{\prime \prime}<0$, the same reasoning gives $f^{\prime \prime}<0$.

Finally, we can provide an application to the relevant family of the Friedman-RobertsonWalker spacetimes, well known in Cosmology. These spacetimes constitute a family of exact solutions of the Einstein equations and represent physically realistic universe models. Since a Friedman-Roberson-Walker is a Robertson-Walker spacetime filled with perfect fluid, which is a dust, then its warping function $f$ satisfies $f^{\prime \prime}<0$ (see, [25, Chap 12]).

Corollary 12 In a Friedman-Robertson-Walker spacetime, any maximal hypersurface is strongly stable.

As a consequence, a complete maximal hypersurface in a Friedman-Robertson-Walker spacetime describes the turn around epoch.

\section{References}

[1] J.A. Aledo, R.M. Rubio, Scalar curvature of spacelike hypersurfaces and certain class of cosmological models for accelerated expanding universes, J. Geom. Phys., 104 (2016), 128-136.

[2] L.J. Alías, A. Romero and M. Sánchez, Uniqueness of complete spacelike hypersurfaces of constant mean curvature in Generalized Robertson-Walker spacetimes, Gen. Relat. Gravit., 27 (1995), 71-84.

[3] L.J. Alías, A. Romero, M. Sanchez, Spacelike hypersurfaces of constant mean curvature in certain spacetimes, Nonlinear Analysis TMA 30 (1997), 655-661.

[4] J.L.M. Barbosa, V Oliker, Spacelike hypersurfaces with constant mean curvature in Lorentz space, Mat. Contemp. 4 (1993), 2744.

[5] J. Barta, Sur la vibration fundamentale d'une menbrane, C.R. Acad. Sci. 204 (1937), $472-473$.

[6] R. Bartnik, Existence of maximal surfaces in asymptotically flat spacetimes, Commun. Math. Phys. 94 (1984), 155-175.

[7] A. Brasil, A.G. Colares, On constant mean curvature spacelike hypersurfaces in Lorentz manifolds, Mat. Contemp., 17 (1999) 99-136.

[8] D. Brill, F. Flaherty, Isolated maximal surfaces in spacetime, Commun. Math. Phys., 50, (1976) 157-165.

[9] E. Calabi, Examples of Bernstein problems for some nonlinear equations, Proc. Symp. Pure Math., 15 (1970), 223-230.

[10] M. Caballero, A. Romero and R.M. Rubio, Constant mean curvature spacelike hypersurfaces in Lorentzian manifolds with a timelike gradient conformal vector field, Class. Quantum Grav., 28 (2011), 145009-145022. 
[11] S.Y. Cheng and S.T. Yau, Maximal spacelike hypersurfaces in the Lorentz-Minkowski spacetime, Ann. Math., 104 (1976), 407-419.

[12] Y. Choquet-Bruhat, Maximal submanifolds and submanifolds with constant mean extrinsic curvature of a Lorentzian manifold, Ann. Scuola Norm. Sup. Pisa, 3 (1976), $361-376$.

[13] Y. Choquet-Bruhat and R. Geroch, Global Aspects of the Cauchy Problem in General Relativity, Commun. Math. Phys., 14 (1969), 329-335.

[14] Y. Choquet-Bruhat, The problem of constraints in General Relativity: solution of the Lichnerowicz equation, Differential Geometry and Relativity, Math. Phys. Appl. Math. 3, D. Reidel, Dordrecht (1976), 225-235.

[15] Y. Choquet-Bruhat, General Relativiy and the Einstein Equations, Oxford Math. Monogr., Oxford Univ. Press, New York, 2009.

[16] D. Fischer-Colbrie and R. Schoen, The structure of complete stable minimal surfaces in 3-manifolds of non-negative scalar curvature, Comm. Pure Appl. Math., 33 (1980), 199-211.

[17] V. Daftardar and N.Dadhich, Gradient conformal Killing Vectors and Exact solutions, Gen. Relativity Gravitation, 26 (1994), 859-868.

[18] Earley, D., Isenb erg, J ., Marsden, J ., Moncrief, V. Homothetic and conformal symmetries of solutions to Einsteins equations, Commun . Math. Phys, (1986) 106-137.

[19] T. Frankel, Applications of Duschek's formula to cosmology and minimal surfaces, Bull. Amer. Math. Soc., 81 (1975), 579-583.

[20] R.P. Geroch, Limits of spacetimes, Comm. Math. Phys. 13 (1969), 180-193.

[21] S. H., Kramer D, M. MacCallum , C. Hoenselaersand, E. Herlt, Exact Solutions of Einsteins Field Equations, Cambridge University Press, Cambridge, 2003.

[22] J. Marsden, J.H. Tipler, Maximal hypersurfaces and foliations of constant mean curvature in General Relativity, Phys. Rep., 66 (1980), 109-139.

[23] S. Nishikawa, On maximal spacelike hypersurfaces in a Lorentzian manifold, Nagoya Math. J., 95 (1984), 117-124.

[24] H. Omori, Isometrics immersions of Riemannian manifolds, J. Math. Soc. Japan, 19 (1967), 205-214.

[25] B. O'Neill, Semi-Riemannian Geometry with Applications to Relativity, Pure Appl. Math., 103, Academic Press, New York, 1983.

[26] M. Rainer and H-J. Schmidt, Inhomogeneous cosmological models with homogeneous inner hypersurface geometry, Gen. Relativity Grav 27 (1995), 1265-1293.

[27] A. Romero, R.M. Rubio, J.J. Salamanca, Complete maximal hypersurfaces in certain spatially open generalized Roberson-Walker spacetimes, R.A.C.S.A.M., (2014), DOI 10.1007/s13398-014-0195-1. 
[28] R.K. Sachs and H. Wu, General Relativity for Mathematicians, Grad. Texts Math., Springer-Verlag, New York, 1977.

[29] R. Schoen, L. Simon and S.T. Yau, Curvature estimates for minimal hypersurfaces, Acta Math., 134 (1975), 275-288.

[30] F.J. Tipler, Causally symmetric spacetimes, J. Math. Phys. 18 (1997), 1568-1573.

[31] F.W. Warner. Foundations of Differentiable Manifolds and Lie Groups, Grad. Texts Math., Springer-Verlag, 1983.

[32] S.T. Yau, Harmonic functions on complete Riemannian manifolds, J. Math. Phys., 28 (1973), 201-228.

[33] J. York, N. Murchadha, Initial value problem of general relativity, Phys. Rev. D, 10 (1974), 428-446.

[34] E. Zafiris, Irreducible decomposition of Einstein's equations in spacetimes with symmetries, Ann. Phys., 263 (1998), 155-178. 